\title{
Homotopy theory in abelian categories
}

\section{Doctoral Thesis}

Author(s):

Kleisli, Heinrich

Publication date:

1962

Permanent link:

https://doi.org/10.3929/ethz-a-000088033

Rights / license:

In Copyright - Non-Commercial Use Permitted 
Prom. Nr. 3055

\section{HOMOTOPY THEORY IN ABELIAN CATEGORIES}

VON DER

EIDGENÖSSISCHEN TECHNISCHEN HOCHSCHULE IN ZÜRICH

ZUR ERLANGUNG DER WÜRDE EINES DOKTORS DER MATHEMATIK

GENEHMIGTE

\section{PROMOTIONSARBEIT}

VORGELEGT VON

HEINRICH KLEISLI

alpl. Math ETI

VON NEDER WENINGEN (2AT)

Referent: Herr Prof. Dr. B. EскMANN

Korreferent: Herr Prof. Dr. E. Splсскек

1962

UNIVERSITY OF TORONTO PRESS, TORONTO, ONT. 


\section{ZUSAMMENFASSUNG}

Der Homotopiebegriff, der von Eckmann und Hilton in der Kategorie der Moduln über einem Ring eingeführt wurde $(3 ; \mathbf{9} ; \mathbf{1 0})$, wird in dieser Arbeit auf allgemeinere additive Kategorien übertragen. Es wird gezeigt, wie die Homotopietheorie der Moduln und insbesondere die Sätze über die Exaktheit der verschiedenen Homotopiesequenzen für derartige abstrakte Kategorien in allgemeiner Form entwickelt werden können.

Abgesehen von der Möglichkeit neuer Anwendungen werden in unserer Verallgemeinerung zwei Ziele angestrebt. Erstens eine vollständig selbstduale Entwicklung der Theorie; zweitens soll der Rahmen so gewählt werden, dass die hergeleiteten Sätze ohne weiteres auch in der Kategorie der Paare gelten. Dabei ist zu beachten, dass in der Kategorie der Paare, wie schon im Falle der Moduln in (3), eine Klasse von Monomorphismen und Epimorphismen ausgezeichnet werden muss.

In der Kategorie der Paare von Moduln bildet nun die erwähnte Klasse ausgezeichneter Morphismen eine "Abelsche Struktur" in Sinne von Heller (8). Deshalb führen wir unsere Verallgemeinerung in einer additiven Kategorie mit Abelscher Struktur (auch kurz Abelsche Kategorie genannt) durch. Es zeigt sich, dass diese Wahl der zugrundegelegten Kategorie sowohl in der Entwicklung der Theorie als auch in bezug auf neue Anwendungen manche Vorteile bietet.

In einem ersten Abschnitt werden die grundlegenden Definitionen, welche mit dem Begriff der Abelschen Kategorie zusammenhängen, formuliert. Ferner werden Beispiele angegeben, teils um die eingeführten Begriffe zu illustrieren, teils um die Überlegungen in den folgenden Abschnitten vorzubereiten.

Im zweiten Abschnitt werden die Grundbegriffe der Homotopietheorie in einer additiven Kategorie $\Omega$ mit Abelscher Struktur entwickelt. In der Kategorie $P \Omega$ der Paare in $\Omega$ wird eine Abelsche Struktur, die nur von derjenigen in $\Re$ abhängt, definiert, und "relative" Homotopiegruppen werden als spezielle Homotopiegruppen der Kategorie $P \Omega$ eingeführt. Der Übergang von der Abelschen Kategorie $\Re$ zur Abelschen Kategorie $P \Omega$ kann wiederholt werden und führt zur Abelschen Kategorie $P^{2} \Re$ der Doppelpaare in $\Re$.

In einem weiteren Abschnitt werden verschiedene Homotopiesequenzen untersucht, nämlich je zwei zueinander duale Homotopiesequenzen eines Paares und eines Tripels. Statt deren Exaktheit an jeder Stelle direkt zu verifizieren, wird eine Methode benützt, bei welcher die Überlegungen in der Kategorie der Paare durchgeführt werden, und bei welcher die Exaktheit aus der bekannten Tatsache folgt, dass die Homologiesequenz einer exakten Sequenz von Komplexen exakt ist. Ferner werden zwei duale FaserungsBegriffe behandelt und die entsprechenden "Exzisionssätze" bewiesen.

Der letzte Abschnitt enthält drei Anwendungen auf spezielle Kategorien. Man erhält dabei spezielle Homotopiebegriffe, nämlich die Homotopie für Moduln über einem Ring, die "schwache Homotopie" für Moduln über einer Algebra, die Kettenhomotopie für Komplexe. 